\title{
ANALYSIS OF LOAD CHARACTERISTICS AND CONTROL OF WATER HYDRAULIC SYSTEM FOR WAIM
}

\author{
Zengmeng ZHANG, Hua ZHOU \\ State Key Laboratory of Fluid Power Transmission and Control \\ Zhejiang University \\ 38 Zheda Road, Hangzhou, 310027 China \\ (E-mail: zzm.zju@gmail.com)
}

\begin{abstract}
The load characteristics and control of water hydraulic system for water-assisted injection molding (WAIM) are investigated through modeling and simulation. WAIM is an innovative process to mold plastic parts with hollow sections, coming with big advantages: high production efficiency and quality at low cost. The load characteristic is complicated owing to physical properties of polymer, distribution of temperature, flow rate and water channel geometry etc. The load pressure and flow rate are investigated with CFD simulation. An accumulator and a supercharger are applied in the water hydraulic system which is modeled in MATLAB and developed in laboratory. A differential pressure control strategy is provided to regulate the water injection pressure by a proportional relief valve. PI control is used and the steady-state error of step response can be reduced close to zero with integral compensation in closed-loop control. Finally, slope control of injection pressure is studied based on the developed system in simulation. The aim of this study is to improve the control process of WAIM based on the new design and differential pressure control. In addition, this provides an application and practice of water hydraulic proportional control technology.
\end{abstract}

\section{KEY WORDS}

Water hydraulics, Water-assisted injection molding, Load characteristic, Pressure control

\section{NOMENCLATURE}

\section{$A$ : Area of piston}

$A_{L}: \quad$ Cross area of internal cavity of mold

$B_{L}: \quad$ Visco-damping coefficient of load

$C_{i p}:$ Inner leakage coefficient

$C_{e p}:$ Outer leakage coefficient

$f:$ Static friction force of piston

$L \quad$ : Length of the cavity-filling polymer

$m$ : Equivalent mass of load

$p$ : Pressure of chamber in cylinder and load

$q$ : Flow rate

$r:$ Radius of the cavity in mold

$T$ : Temperature of polymer

\author{
$x$ : Displacement of piston \\ $\beta_{e}:$ Bulk modulus \\ $\eta$ : Coefficient of polymer viscosity \\ $\lambda:$ Heat transfer coefficient \\ $\omega:$ Frequency of control link
}

\section{INTRODUCTION}

Water-assisted injection molding (WAIM) is a novel way to mold hollow plastic parts. When the water is injected into the molten regions of a part, cavities are generated and polymer melt is displaced to form a hollow core. It is similar to gas-assisted injection molding (GAIM), but has the advantages such as short 
cooling cycle time, high quality of products and low cost, for the reason of the difference between water and gas[1 2]. Especially, WAIM can produce parts with larger void spaces or longer hollow sections than GAIM. It brings the applications of WAIM to a broad field of elongated parts such as automotive industry, office equipment, medical products and building materials [3]. The load characteristic is complicated and coupling with the flow rate and load pressure. The cavity filling process is investigated in a great many researches for GAIM but few in WAIM. The general method used in GAIM simulation is Hele-Shaw model and suitable to thin wall molding perfectly. However there is high Re region in the field of water and the thin-wall model is not suitable to large parts molding. It is necessary to involve turbulent calculation to model the couple relationship between load pressure and flow rate. The load characteristics are the basis of modeling and control of water hydraulic system for WAIM.

In recent years, water hydraulic technology became a hot research area with an increase of concerns for global environment problems and mineral oil. Water hydraulics has some general benefits (e.g., environmental friendliness, non-flammability, easy availability, inexpensiveness) compared to oil hydraulics. The high bulk modulus, constant viscosity and low temperature-induced drift are praised highly in water hydraulic proportional control technique. However, the pressure impact in water hydraulic system increases with the high bulk modulus. Especially, the disturbance of flow fluctuation is serious in process of water injection and causes uncontrollable status frequently. Because of the seal and lubrication in components, the nonlinearity in water hydraulic control systems is much greater than oil systems. The static friction and leakage flow will cause large dead band and hysteresis as disturbance additionally $[4,5]$. Hence, the precision of the pressure control of water hydraulic system is lower relatively compared with conventional oil hydraulics. This motivates to apply closed-loop control to improve the performance and capability to resist disturbance. In this paper, load characteristics and water injection process of WAIM are analyzed, based on which a new water hydraulic system with differential proportional pressure control and compound control of pressure and flow rate is developed.

\section{LOAD CHARACTERISTICS}

\section{Analysis of Water Injection Process}

The whole work cycle in WAIM mainly contains five steps: polymer melt injection, water injection, maintaining pressure, drainage and part ejection, as shown in Fig. 1 [6]. To avoid turbulence in the area of the water injector, the recommendation is to adjust the pressure profile starting with low pressure and then increasing to higher pressure. Large flow is supplied at the same time. The process of WAIM is intermittent and the injection stage takes very short time, typically only one or two seconds, which is a moment within the whole work cycle. The pressure is maintained after water injection and the flow rate decreases from the total quantity to a very small level to supply the melt shrinkage. The problem about energy saving is how the large-flow injection assorts with the small-flow pressure maintaining in the whole cycle of WAIM.

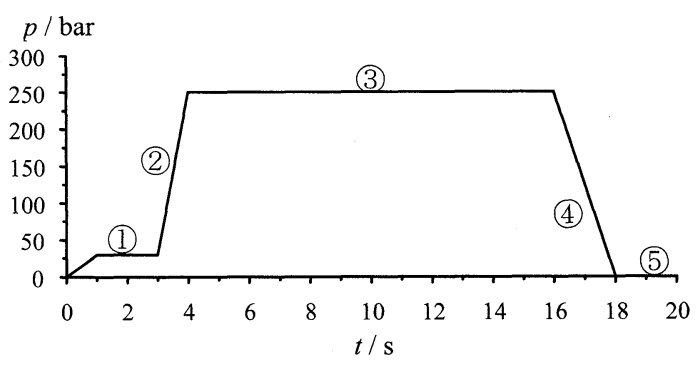

Figure 1 Load pressure profile of WAIM

Certain parameters such as water injection time, pressure profile and temperature of the injected water play an important role in achieving high quality residual wall. The necessary water injection pressure depends on the flow resistance of the plastic material and the part geometry. When injected into the parts, water cools the polymer melt and increases the viscosity. The leading edge of the water forms a solid boundary or highly viscous membrane. The membrane forces molten material forward, instead of the polymer forcing the water to the side [7]. The process of WAIM involves the dynamic interaction of two very different materials (polymer melt and filling water), and strongly depends on the temperature due to viscosity-temperature performance of polymer. The necessary water injection pressure depends on the flow resistance of the plastic material and the part geometry. So the simplification of the cavity filling process is needed to get a linear model of load pressure and flow rate.

\section{CFD Simulation}

The viscosity of the load is considered. A Cross-Arrhenius model is suitable to model the viscosity characteristic of polymer melt cavity filling flow in molding injection. The equation is

$$
\eta(\dot{\gamma}, T, p)=\frac{\eta_{0}(T, p)}{1+\left(\eta_{0} \dot{\gamma} / \tau^{*}\right)^{1-n}}
$$

where

$$
\eta_{0}(T, p)=B \exp \left(T_{B} / T\right) \exp (\beta p)
$$

Under the condition that temperature drops to a point $T<T_{g}+100 \mathrm{~K}$, The Cross-WLF model shall be used as 


$$
\eta_{0}(T, p)=\eta_{g} \exp \frac{-C_{1}\left(T-T_{g}\right)}{C_{2}+T-T_{g}} \exp (\beta p)
$$

Here $B, T_{B}, \beta, \tau^{*}, C_{1}$ and $C_{2}$ are parameters of the Cross model and are quite different between polymers. The apparent viscosity $\eta$ is influenced by temperature, shear stain rate and pressure in injection. The load pressure $p$ can be given as

$$
p=2 L \eta(\dot{\gamma}) \dot{\gamma} / r
$$

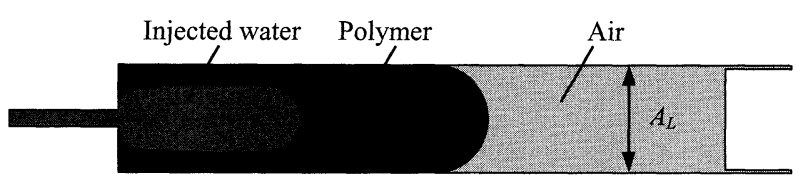

Figure 2 The calculation field of CFD simulation

There are three phases: water, polymer melt and air as shown in Fig. 2. A $k-\omega$ turbulence model is used in the field of water and laminar in polymer melt. The effective heat transfer coefficient $\lambda_{\text {eff }}$ and turbulent viscosity $\mu_{t}$ are written as

$$
\begin{aligned}
& \lambda_{e f f}=\lambda+\frac{C_{p} \mu_{t}}{\operatorname{Pr}_{t}} \\
& \mu_{t}=\alpha^{*} \frac{\rho k}{\omega}
\end{aligned}
$$

where $C_{p}, \operatorname{Pr}_{t}, \alpha^{*}, k, \omega$ are $k-\omega$ model parameters. The flow front is solved by VOF model and PISO coupling format of velocity and pressure is applied in this unsteady calculation. Results of volume fraction, temperature and ratio of turbulent viscosity are shown in Fig. 3. These show small residual wall gained with simulation close to the experiment results $[6,8,9]$. The maximum of turbulent viscosity ratio $\max \left(\mu_{t} / \mu\right)=$ $1.38 \times 10^{3}$ is in a correct range. Temperature changes greatly on the interface between water and polymer melt. Results of the coupling between load pressure and flow rate are shown in Fig. 4.

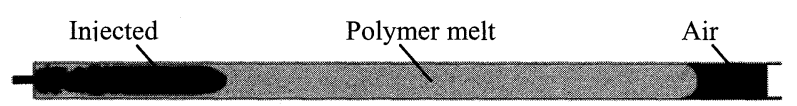

(a) Volume fraction of phases $(t=0.2 \mathrm{~s})$

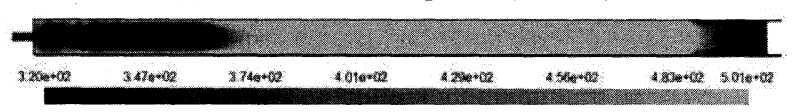

(b) Static temperature $(t=0.2 \mathrm{~s})$

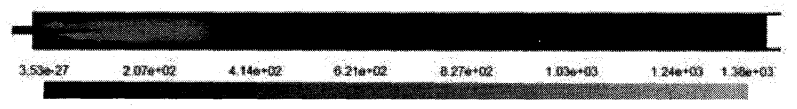

(c) Ratio of turbulent viscosity $\left(\mu_{t} / \mu\right)(t=0.2 \mathrm{~s})$

Figure 3 Cavity filling flow simulation for WAIM

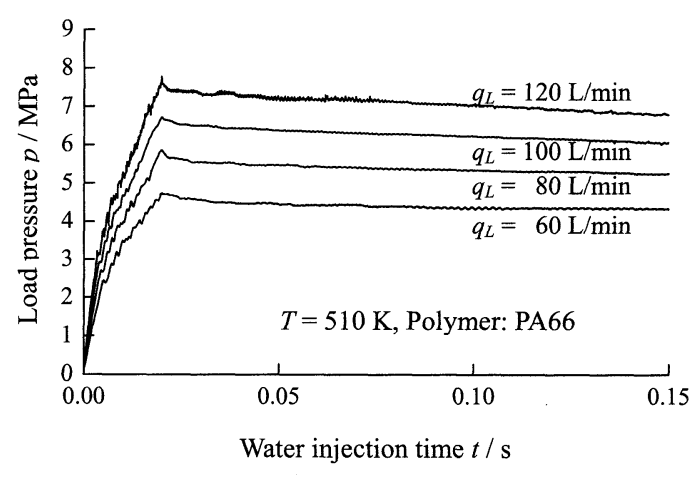

Figure 4 CFD simulation results of load characteristic

\section{Linear Model}

The pressure changes according to the flow rate as in Fig. 4 and the approximate equation can be written as

$$
p=\frac{B_{L}}{A_{L}^{2}} q_{L}
$$

where $B_{L}$ is the equivalent visco-damping coefficient of load and can be given from the results of CFD simulation or WAIM experiments.

Considering the load inertia, elasticity and viscosity, the load pressure can be estimated as transfer function

$$
G_{L}(s)=\frac{P}{Q_{L}}=\frac{m_{L} s^{2}+B_{L} s+k_{L}}{A_{L}^{2} s}
$$

There are a restrictor of water injector and a throttle valve linearized as

$$
Q_{L}=k_{t L}\left(P_{L}-P\right)
$$

where $k_{t L}$ is the pressure gain on flow rate of the throttle valve and injector. By Eq. (8) and (9), we obtain that

$$
G_{L}^{\prime}(s)=\frac{P_{L}}{Q_{L}}=\frac{m_{L} s^{2}+B_{L}^{\prime} s+k_{L}}{A_{L}^{2} s}
$$

where $B_{L}^{\prime}=B_{L}+A_{L}{ }^{2} / k_{t L}$. The linear control model of the system can be built and simulated with this assumption of visco-elastic load.

\section{PRESSURE CONTROL}

The simple water hydraulic system including main components is shown in Fig. 5. The equilibrium point at $\left\{p_{A 0}^{\prime}, p_{A 0}, p_{a 0}, V_{a 0}, q_{A 0}, p_{B 0}, p_{L 0}, p_{0}, q_{L 0}\right\}$ is investigated linearizing the accumulator, pressure cylinder, relief valve and throttle valves.

Here only discharge process of the accumulator is investigated and this process is fast and suggested as 


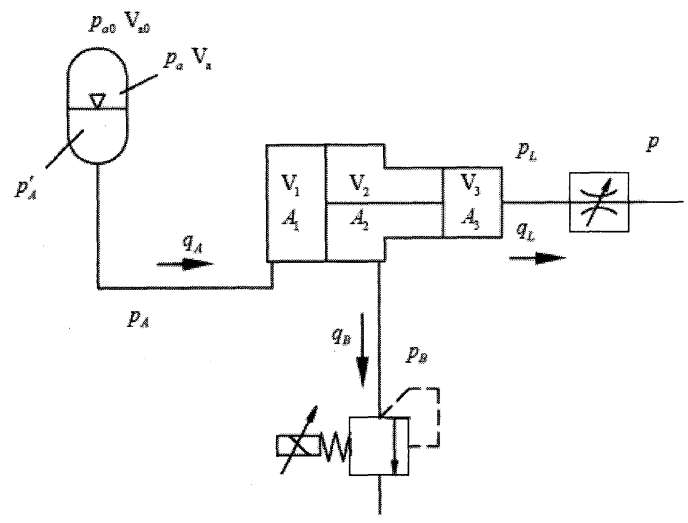

Figure 5 Main components and parameters

adiabatic. Fluid is incompressible in contrast with gas. And no turbulent flow appears in the accumulator [10]. Based on these assumptions and linearization of compressing process of gas, the linear model of accumulator with inlet characteristic can be written as

$$
G_{A}(s)=\frac{P_{A}}{Q_{A}}=-\frac{m_{a} s^{2}+B_{a} s+k_{a}}{A_{a}^{2} s}
$$

where $k_{a}=n_{a} p_{a 0} A_{a}^{2} / V_{a 0}$ and $B_{a}=B_{a 0}+A_{a}^{2} / k_{t A}$. Here $n_{a}, A_{a}, B_{a 0}$ and $k_{t A}$ respectively is polytropic exponent, area of cross section, visco-damping coefficient of liquid part and inlet pressure gain on flow rate of the accumulator, as well as $p_{a 0}$ and $V_{a 0}$ are the initial value of the pre-charge gas. The continuity of water flow and force balance equations for pressure cylinder can be written as

$$
\begin{gathered}
p_{A} A_{1}=p_{B} A_{2}+p_{L} A_{3}+m \ddot{x}+B \dot{x}+f \\
q_{A}=A_{1} \dot{x}+C_{i p_{1}}\left(p_{A}-p_{B}\right)+C_{e p_{1}} p_{A}+\frac{V_{1}}{\beta_{e}} \dot{p}_{A} \\
q_{L}=A_{3} \dot{x}+C_{i p_{2}}\left(p_{B}-p_{L}\right)-C_{e p_{2}} p_{L}-\frac{V_{3}}{\beta_{e}} \dot{p}_{L}
\end{gathered}
$$

Non-external leakage consideration and Laplace transformation of these three equations yield

$$
\begin{aligned}
& P_{A} A_{1}=P_{B} A_{2}+P_{L} A_{3}+m s^{2} X+B s X+F \\
& Q_{A}=A_{1} s X+C_{i p_{1}}\left(P_{A}-P_{B}\right)+\frac{V_{1} s}{\beta_{e}} P_{A} \\
& Q_{L}=A_{3} s X+C_{i p_{2}}\left(P_{B}-P_{L}\right)-\frac{V_{3} s}{\beta_{e}} P_{L}
\end{aligned}
$$

In general, The transfer function of proportional relief valve can be regarded as a three-order system like

$$
\frac{P_{B}}{U}=\frac{k_{r}}{\left(\frac{s^{2}}{\omega_{r 2}^{2}}+\frac{2 \xi_{r}}{\omega_{r 2}} s+1\right)\left(\frac{s}{\omega_{r 1}}+1\right)}
$$

This proportional relief valve is the key component of the system. The accuracy is poor and need closed-loop control to improve performance. But the nonlinearity of hysteresis may lead to oscillation and compensation methods will be investigated in the future work.

Let

$$
\begin{aligned}
& G_{1}=\frac{V_{1} s}{\beta_{e}}+C_{i p_{1}} \\
& G_{2}=\frac{V_{2} s}{\beta_{e}}+C_{i p_{2}}
\end{aligned}
$$

Reduction the equations can yield the control structure of the model of whole system and built in MATLAB. The main parameters set in simulation are shown in Tab. 1 and the Bode diagram is shown in Fig. 6.

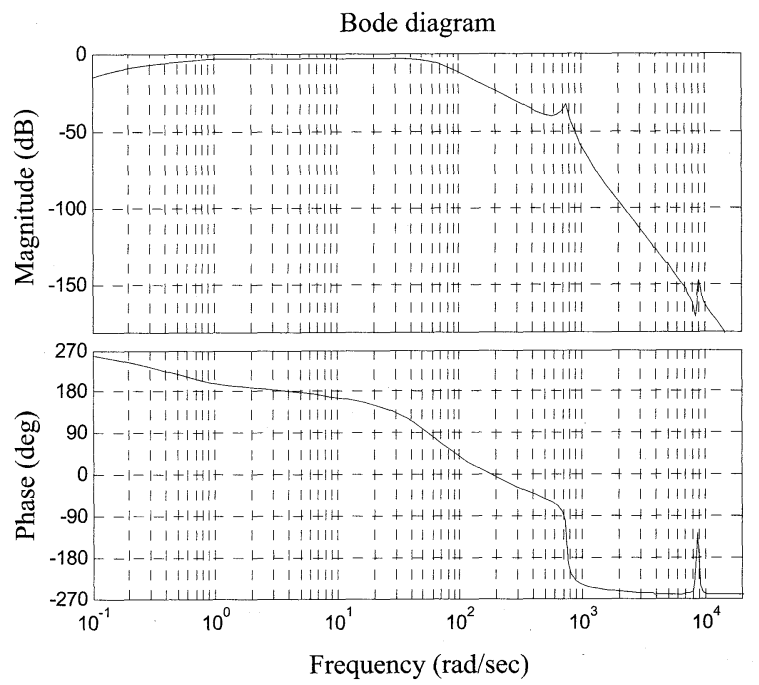

Figure 6 Bode diagram of the system

Table 1 Main parameters for simulation

\begin{tabular}{cccc}
\hline Parameter & Value & Parameter & Value \\
\hline$k_{a}$ & $3.8 \times 10^{5} \mathrm{~N} / \mathrm{m}$ & $B$ & $39 \mathrm{~kg} / \mathrm{s}$ \\
$B_{a}$ & $1422 \mathrm{~kg} / \mathrm{s}$ & $m_{L}$ & $3.2 \mathrm{~kg}$ \\
$A_{a}$ & $0.015 \mathrm{~m}^{2}$ & $f$ & $90 \mathrm{~N}$ \\
$\beta_{e}$ & $1.6 \times 10^{9} \mathrm{~Pa}$ & $B^{\prime}{ }_{L}$ & $6 \times 10^{4} \mathrm{~kg} / \mathrm{s}$ \\
$A_{1}$ & $0.012 \mathrm{~m}^{2}$ & $A_{L}$ & $0.002 \mathrm{~m}^{2}$ \\
$A_{2}$ & $0.006 \mathrm{~m}^{2}$ & $\omega_{r 2}$ & $40 \mathrm{rad} / \mathrm{s}$ \\
$A_{3}$ & $0.006 \mathrm{~m}^{2}$ & $\xi_{r}$ & 0.7 \\
$m$ & $31 \mathrm{~kg}$ & $\omega_{r 1}$ & $320 \mathrm{rad} / \mathrm{s}$ \\
\hline
\end{tabular}

There are two differential links in the open-loop. Furthermore, the load pressure may not follow the input 
signal. This is because there is accumulator and the back pressure of cylinder is controlled by relief valve. If no feedback was supplied to the input to tune the back pressure, such a constant command signal set, the accumulator would charge or discharge to change pressure to a new equilibrium point.

Based on the static characteristic of force balance in pressure cylinder, a local feedback strategy for the differential pressure control is defined as

$$
U^{\prime}=\frac{k_{f a} P_{A} A_{1}-U A_{3}}{A_{2}}
$$

where $k_{f a}$ is the gain of pressure transducer. The pressure of accumulator feedbacks to make the controller response following the pressure decrease and the load pressure be consistent with input. The structure of pressure control is improved and this simple controller is reliable, however, without any other compensation and load pressure feedback, a large error is obvious by reason of the resistance of throttle valves, injector, pipes etc.

A '0-type' system is formed in the open-loop control with correction by Eq. (21). Integral compensation is necessary to be applied in the closed-loop controller. The gain $k_{I}$ of the integral part is requested as

$$
k_{I}<\omega_{r 2}
$$

for the reason of the requirement of stability. As shown in Fig. 7, the steady-state error of step response can be reduced close to zero under this closed-loop control in theory.

For slope control of the pressure when water is injected, steady-state error is decreased while $k_{I}$ is set to a large value as shown in Fig. 8. Nevertheless the stability and the capacity of resisting disturbance become poor.

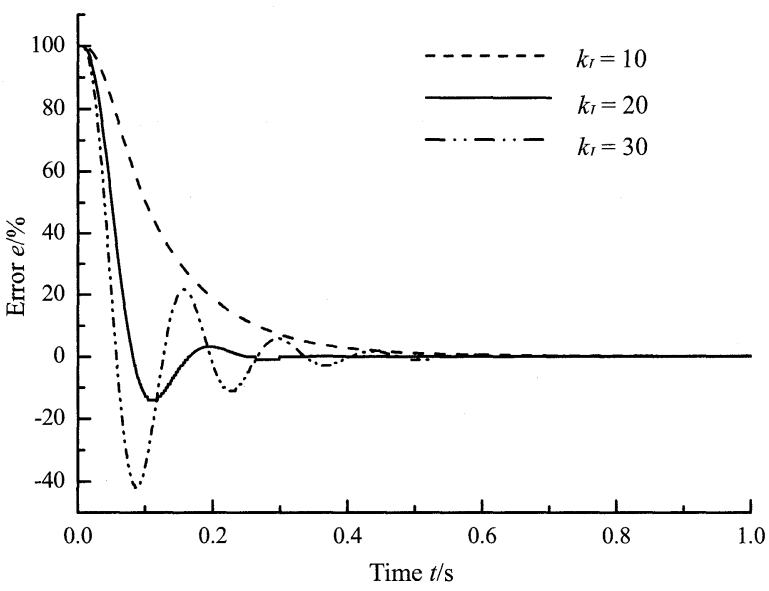

Figure 7 Step-response error with closed-loop control
The load viscosity and elasticity change largely than inertia in WAIM from different polymers. The flow rate is greatly influenced by load characteristics as shown in Fig 9. And the load viscosity has relatively large influence

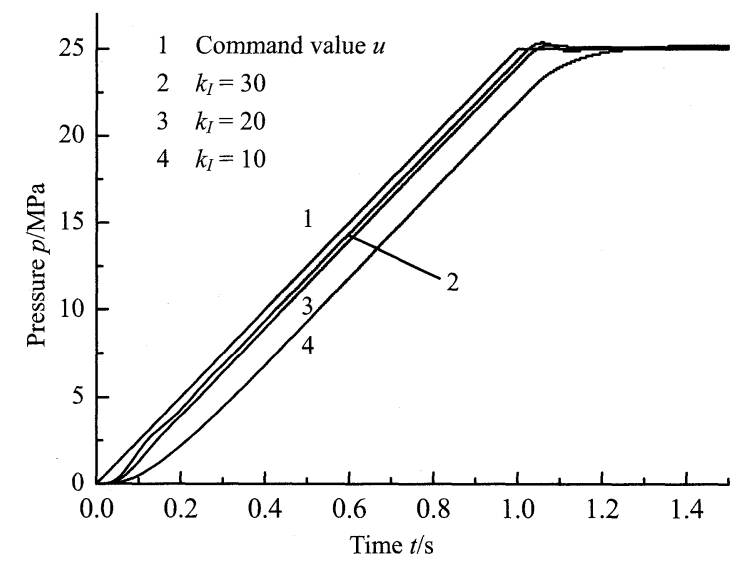

Figure 8 Slope control for various gain of integrator

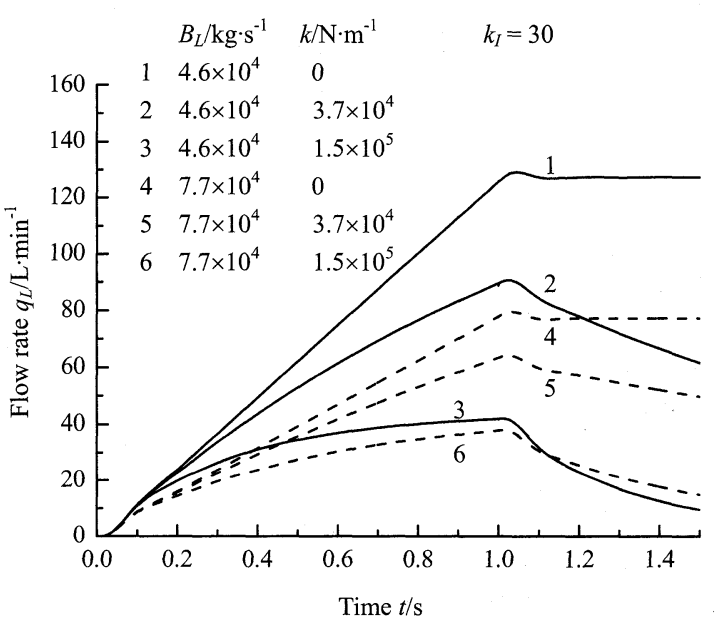

Figure 9 Simulation results of load flow rate

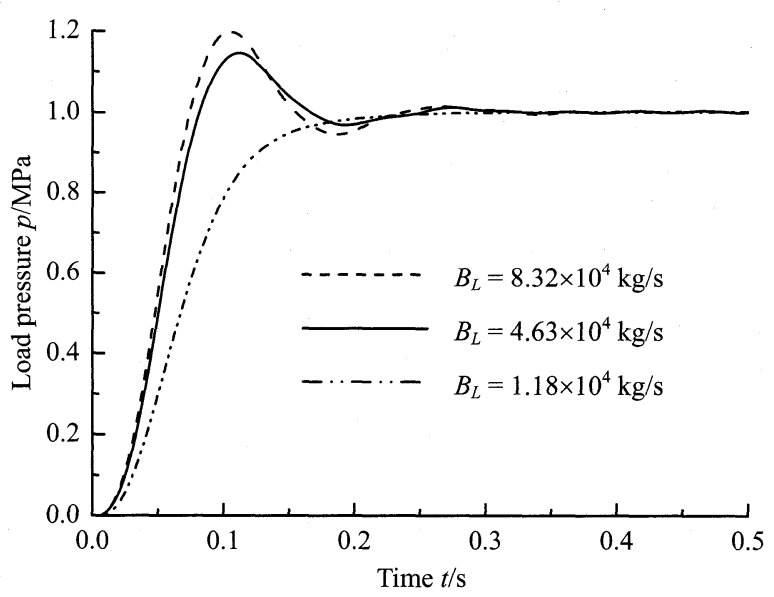

Figure 10 Step responses for different load viscosity 
on pressure control. Also the slopes tend to be shallow along with the increase of elasticity. The curves may be flat as pressure becomes constant if the elasticity is small. Large elasticity makes flow rate decrease fast when the pressure is maintained. The serials of step responses for closed-loop control with different load viscosity are shown in Fig. 10. The damping ratio of the system increases as the visco-damping coefficient of the load decreases. This illustrates that high viscosity of the polymer melt makes the injection pressure fluctuate when a large input signal change occurs. This shall appear on the start and end of water injection. So a low slope or gentle start should be used to increase injection pressure when molding polymer with high viscosity.

\section{CONCLUSIONS}

In this paper, we presented a new control strategy with differential pressure control and energy saving applying accumulator. Through analysis of load characteristic and modeling of the pressure control system, we developed the closed-loop controller.

The differences between polymer melts with load characteristic are great, especially the viscosity and elasticity. The coupling between load pressure and flow rate can be illustrated as linear relationship at aspect of viscosity and an assumption of linear model for WAIM load characteristic is provided. High viscosity leads to large oscillation and instability. The gain of the integrator should be reduced with high-viscosity polymer melt and a low slope or gentle start of command signal should be used to increase injection pressure when molding high-viscosity polymer.

The linear model of the system is built and a '0-type' system is discovered by the feedback of pressure at the accumulator. Based on the closed-loop control with integral compensation, the steady-state error can be reduced close to zero for step response besides the disturbance of nonlinear factors and load fluctuation. Large gain of integrator can be tuned for slope pressure control in WAIM to reduce the error. However, this may result in oscillation of pressure due to nonlinearity of water hydraulic valves. A fitting value of the gain shall be tested in the future experiments. The differential pressure control with pressure piston and accumulator is suitable to the work characteristic of WAIM.

Further research is carried out experimentally. The coupling of load pressure and flow rate and pressure control performance will be measured and investigated.

\section{ACKNOWLEDGMENTS}

The authors acknowledge the support of the National Natural Science Foundation of China (NSFC), Grant 50775199. And the Science and Technology Plan Project of Zhejiang province, Grant 2007C21057, is also acknowledged.

\section{REFERENCES}

1. Mikell, K., Water Injection Molding Makes Hollow Parts Faster, Plastics Technology, 2002, 48-4, pp. $42-47$

2. Walter, M., Andre, B. and Tim Pohl,. A Comparison of Gas and Water Injection Moulding of Hollow Articles by Fluid-Assisted Injection, Kunststoffe Plast Europe, 1999, 89-9, pp.18-20

3. Walter, M., Tim, J. and Andre, B., WIT-En Route to Series Production: First Industrial Application of the Water Injection Technique, Kunststoffe Plast Europe, 2001, 91-3, pp.37-39

4. TAKAHASHI, H., ITO, K., IKEO, S., Application of Adaptive Controller to Water Hydraulic Servo Cylinder, Proceedings of the 6th JFPS International Symposium on Fluid Power, TSUKUBA, November, 2005, pp.432-436

5. Finn Conrad. Trends in Design of Water Hydraulics Motion Control and Open-Ended Solutions, Proceedings of the 6th JFPS International Symposium on Fluid Power, 2005, pp.420-431

6. Rainer Protte, Hartmut Bangert, Chris Cooper and Peter Hoeck, Water-Assist Injection Molding - An Innovative Process Technology for Productivity Improvement: Developments in Processing, Equipment and Materials, SPE Antec Proceedings, 2003, pp.404-408

7. Shih-Jung Liu and Shih-Po Lin, Study of 'Fingering' in Water Assisted Injection Molded Composites, Composites Part A: Applied Science and Manufacturing, 2005, 36, pp.1507-1517

8. Shih-Jung Liu and Yen-Shou Chen, The Manufacturing of Thermoplastic Composite Parts by Water-assisted Injection-Molding Technology, Composites Part A: Applied Science and Manufacturing, 2004, 35, pp.171-180

9. LIU, S-J and LIN, C-H., An experimental study of water- assisted injection molding of plastic tubes with dimensional transitions, Journal of Reinforced Plastics and Composites, 2007, 26-14, pp.1441-1454

10. QUAN, LX, and KONG, XD, Theory and Experiment of Accumulator Absorbing Pressure Pulsation without Regard to its Entrance Characteristics. Chinese Journal of Mechanical Engineering, 2007, 43-9, pp.28-32, (In Chinese) 